\title{
Maternal and early years vitamin supplementation- evaluating the effectiveness of an Inter-Professional Learning workshop in raising awareness
}

\author{
B. Getty, K. Farren, P. Douglas, L. K. Pourshahidi, M. A. Kerr, R. K. Price and H. McCarthy \\ School of Biomedical Sciences, University of Ulster, Coleraine, Co Londonderry, UK, BT52 ISA
}

There are recognised inconsistencies in maternal and childhood diet and health, between disadvantaged groups and the general population $^{(1)}$. To address this, the National Institute for Health and Clinical Excellence (NICE) have published public health guidance for health professionals working with these groups, including specific recommendations for vitamin and mineral supplementation throughout pregnancy and early life. Sources of information for the public, on vitamin supplementation include, medical, nursing, pharmacy, nutrition and dietetic professionals however the level of awareness among these groups have been questioned. Inter-Professional Learning (IPL) workshops enable information to be shared and explained within different health professional groups $^{(2)}$. Targeting early career professionals and students with inter-professional education has been advocated as an effective teaching and learning strategy. The aim of this study was to evaluate the effectiveness of an IPL workshop, in relation to improved knowledge of maternal and early years vitamin requirements and supplementation

A 3 hour IPL workshop was utilised to deliver education on maternal and early years vitamin requirements and supplementation to a sample of final year and postgraduate students (n93). Students worked in mixed professional groups of Dietetics, Nutrition and Pharmacy. Data were collected by an anonymous questionnaire at the beginning and end of the workshop. The same questions were asked at both timepoints but in a different order, to explore changes in student's knowledge of maternal and childhood vitamins. All questions were multiple choice, with one correct answer for all knowledge base questions. An arbitrary score was assigned to each knowledge based question with a maximum score of 7 . Median scores pre and post workshop were compared for the entire cohort using Wilcoxon Signed Rank test. Median scores were additionally compared at the pre and post workshop timepoints to identify differences between the courses using Kruskal-Wallis followed by Mann-Whitney U test to identify the source of any statistically significant findings, and within courses using Wilcoxon Signed Rank test.

Table 1. Median knowledge scores by course at the pre and post workshop timepoints

\begin{tabular}{|c|c|c|c|c|c|c|c|c|c|c|c|}
\hline & \multirow{2}{*}{\multicolumn{2}{|c|}{$\begin{array}{l}\text { Human Nutrition } \\
\text { (BSc) } \\
N 20\end{array}$}} & \multirow{2}{*}{\multicolumn{2}{|c|}{$\begin{array}{l}\text { Food and Nutrition } \\
\text { (BSc) } \\
N 30\end{array}$}} & \multirow{2}{*}{\multicolumn{2}{|c|}{$\frac{\text { Dietetics (BSc) }}{N 15}$}} & \multirow{2}{*}{\multicolumn{2}{|c|}{$\frac{\text { Pharmacy (MPharm) }}{N 23}$}} & \multirow{2}{*}{\multicolumn{2}{|c|}{$\frac{\text { Dietetics (MSc) }}{N 5}$}} & \multirow[b]{3}{*}{$P^{1}$} \\
\hline & & & & & & & & & & & \\
\hline & Median & $\overline{\mathrm{IQR}}$ & Median & $\overline{\mathrm{IQR}}$ & Median & $\overline{\mathrm{IQR}}$ & Median & $\overline{\mathrm{IQR}}$ & Median & $\overline{\mathrm{IQR}}$ & \\
\hline Pre & $4 \cdot 00$ & $1 \cdot 00$ & $4 \cdot 00$ & $1 \cdot 00$ & 5.00 & $2 \cdot 00$ & 3.00 & $1 \cdot 75$ & $4 \cdot 00$ & $2 \cdot 25$ & $0 \cdot 001$ \\
\hline Post & $6 \cdot 00$ & $1 \cdot 00$ & $6 \cdot 00$ & $2 \cdot 00$ & $7 \cdot 00$ & $1 \cdot 00$ & $7 \cdot 00$ & $1 \cdot 00$ & $6 \cdot 00$ & $1 \cdot 00$ & $0 \cdot 296$ \\
\hline
\end{tabular}

${ }^{1}$ Values compare between courses at the pre and post timepoints using Kruskal-Wallis; $P<0 \cdot 05$ significant.

Overall the workshop proved effective with a significant increase in knowledge scores for the full cohort (pre-IPL median 4.00; IQR 1.00: post-IPL median 6.00; IQR 1.00: $P<0.001)$ and within courses $(P<0.05$ for all courses $)$. Before the workshop, Pharmacy students scored significantly lower than all other courses except MSc Dietetic $(P<0.05)$. Following the workshop there was no significant difference in scores between the courses. The results of this evaluation support the IPL workshop approach as an effective learning environment for nutrition related healthcare topics with key learning demonstrated. Future work should explore the longer term impact of this learning experience on knowledge and practice for both students and early career professionals.

1. National Institute for Health and Clinical Excellence (2008), PH11 Maternal and Child Nutrition, http://www.nice.org.uk/guidance/PH11 (Accessed on $27 / 1 / 14)$.

2. Health Sciences and Practice Subject Centre (2011) Developing Inter-professional education in health and social care course in the UK. London, Health Sciences and Practice Subject Centre. 